\title{
Dexamethasone administration during definitive radiation and temozolomide renders a poor prognosis in a retrospective analysis of newly diagnosed glioblastoma patients
}

Lisa B. E. Shields ${ }^{1,2}$, Brent J. Shelton ${ }^{3}$, Andrew J. Shearer ${ }^{3}$, Li Chen ${ }^{3}$, David A. Sun ${ }^{1,2}$, Sarah Parsons ${ }^{2,4}$,

T. David Bourne ${ }^{2}$, Renato LaRocca ${ }^{2,4}$ and Aaron C. Spalding ${ }^{2,45^{*}}$

\begin{abstract}
Background: Dexamethasone (DXM) is commonly used in the management of cerebral edema in patients diagnosed with glioblastoma multiforme (GBM). Bevacizumab (BEV) is FDA-approved for the progression or recurrence of GBM but has not been shown to improve survival when given for newly diagnosed patients concurrently with radiation (RT) and temozolomide (TMZ). Both DXM and BEV reduce cerebral edema, however, DXM has been shown to induce cytokine cascades which could interfere with cytotoxic therapy. We investigated whether DXM would reduce survival of GBM patients in the setting of concurrent TMZ and BEV administration.

Methods: We reviewed the treatment of all 73 patients with GBM who received definitive therapy at our institution from 2005 to 2013 with RT (60 Gy) delivered with concurrent daily TMZ $\left(75 \mathrm{mg} / \mathrm{m}^{2}\right)$. Of these, 34 patients also were treated with concurrent BEV (10 mg/kg every two weeks). Patients received adjuvant therapy (TMZ or TMZ/Bev) until either progression, discontinuation due to toxicity, or 12 months after radiation completion. All patients who had GBM progression with TMZ were offered BEV for salvage therapy, with 19 (56 \%) receiving BEV.

Results: With a median follow-up of 15.6 months, 67 (91.8\%) patients were deceased. The OS for the entire cohort was 15.9 months, while the PFS was 7.7 months. The extent of resection was a prognostic indicator for OS ( $p=.0044)$. The median survival following gross tumor resection (GTR) was 22.5 months, subtotal resection (STR) was 14.9 months, and biopsy was 12.1 months. The addition of BEV to TMZ with RT was borderline significantly associated with increased PFS (9.4 vs. 5.1 months, $p=0.0574$ ) although was not significantly associated with OS (18.1 vs. 15.3 months respectively, $p=0.3064$ ). In patients receiving TMZ, DXM use concurrent with RT was a poor prognostic indicator of both OS (12.7 vs. 22.6 months, $p=0.003$ ) and PFS (3.6 vs. 8.4 months, $p<0.0001$ ). DXM did not reduce OS in patients who received TMZ and BEV concurrently with $\mathrm{RT}$ ( 22.9 vs 22.8 months, $p=0.4818$ ). On multivariable analysis, DXM use predicted an unfavorable OS hazard ratio $(\mathrm{HR})=1.72, p=0.045)$.
\end{abstract}

Conclusions: Our results with TMZ, BEV, and RT are similar to previous studies in terms of PFS and OS. DXM use during RT with concurrent TMZ correlated with reduced OS and PFS unless BEV was administered.

Keywords: Radiation, Oncology, Dexamethasone, Bevacizumab, Temozolomide, Glioblastoma multiforme, Radiation, Survival

\footnotetext{
* Correspondence: Aaron.Spalding@nortonhealthcare.org

${ }^{2}$ The Brain Tumor Center, Norton Healthcare, Louisville, KY, USA

${ }^{4}$ The Norton Cancer Institute, Norton Healthcare, Louisville, KY, USA

Full list of author information is available at the end of the article
}

(c) 2015 Shields et al. Open Access This article is distributed under the terms of the Creative Commons Attribution 4.0 International License (http://creativecommons.org/licenses/by/4.0/, which permits unrestricted use, distribution, and reproduction in any medium, provided you give appropriate credit to the original author(s) and the source, provide a link to the Creative Commons license, and indicate if changes were made. The Creative Commons Public Domain Dedication waiver (http://creativecommons.org/publicdomain/zero/1.0/) applies to the data made available in this article, unless otherwise stated. 


\section{Introduction}

The treatment of patients with GBM poses a challenge as the median patient survival rate after diagnosis is between 12 months with radiation alone and $141 / 2$ months with radiation and TMZ [1]. Several factors play a role in the prognosis of these patients, including age, preoperative performance status assessed by the Karnofsky Performance Scale, and extent of resection [2-5]. Resection of $98 \%$ or more of the tumor has been shown to prolong survival in patients with GBM [4]. Furthermore, residual tumor enhancement by immediate postoperative MRI portends a significant reduction in survival as patients with a residual tumor postoperatively had a 6.595-times higher risk of death in comparison to patients without a residual tumor [2].

TMZ has been shown to improve OS and PFS when given concurrently with RT in newly diagnosed GBM patients [1, 6-9]. Additionally, adjuvant TMZ after completion of RT is a positive prognostic indicator of improved OS and PFS [1, 7-9]. As an alkylating agent, TMZ induces $\mathrm{O}^{6}$-methylguanine DNA lesions leading to apoptosis of tumor cells through mitochondrial activation of caspases in a Bcl-2 dependent mechanism [10]. In vitro, DXM protects GBM cells from TMZ-induced apoptosis by inhibition of caspase cleavage and alteration of bcl-2 levels [11]. Methylation status of the methylguanine methyl transferase gene (MGMT) promoter has been shown to be the strongest predictor for outcome and benefit from TMZ chemotherapy [8]. Tumors that do not express MGMT are more susceptible to chemotherapy with alkylating drugs, while patients with methylated MGMT promoter treated with TMZ and radiotherapy have long-term PFS [8].

DXM is commonly used in the management of GBM patients to treat intracranial edema and to control neurological symptoms, although there are no randomized trials of DXM investigating effects on survival [12]. While DXM may alleviate the negative symptoms that accompany GBM, patients are often encouraged to taper their use of DXM due to the side effects of hyperglycemia, osteoporosis, myopathy, weight gain, and immunosuppression [13]. DXM use may be reduced through administration of vascular endothelial growth factor (VEGF) inhibitors to minimize brain edema [12]. BEV is a monoclonal antibody that inhibits VEGF and may have a corticosteroid-sparing effect in patients with recurrent GBM [12].

We report here the OS and PFS of 73 newly diagnosed GBM patients with a focus on the effect of DXM with and without BEV. This cohort consisted of two initial regimens receiving identical RT: 1) concurrent and adjuvant TMZ, and 2) concurrent and adjuvant TMZ and $\mathrm{BEV}$. This allowed us to study the interaction of DXM, TMZ, and BEV on the OS and PFS of newly diagnosed GBM patients.

\section{Methods}

Under an institutional IRB-approved protocol and in compliance with the Helsinki Declaration, we retrospectively reviewed the treatment of 73 patients with GBM who received 30 fractions of simultaneous integrated boost IMRT delivered either with concurrent and adjuvant BEV and TMZ (combined, $n=34$ ) or TMZ alone (standard, $n=39$ ). This study included all patients at one center who were diagnosed with a GBM between 2005-2013. Our cohort spans an era prior to and after the reporting of RTOG 0825 and AVAGlio studies [14-16]. Patients prior to these studies received the combined arm while those after received standard therapy with BEV offered as salvage therapy in accordance with FDA approval and then possible BEV at relapse.

The extent of resection (gross total, subtotal, or biopsy only) was determined on 24-h postoperative MRI by a radiologist blinded to the neurosurgeon's perspective. Preoperative and postoperative MRI scans were combined with the radiation planning CT scan. A MRI scan with gadolinium (GAD) was performed before initiation of adjuvant therapy and every subsequent three months. Radiation consisted of 30 identical fractions delivered once daily five times per week. The planning target volume prescribed 60 Gy (PTV60) received 2 Gy daily to the tumor bed or residual tumor delineated with $\mathrm{T} 1+\mathrm{GAD}$ plus $1.5 \mathrm{~cm}$ margin while the planning target volume prescribed 54 Gy (PTV54) received 1.8 Gy to the T2 FLAIR $+2.5 \mathrm{~cm}$. The combined regimen consisted of concurrent TMZ $\left(75 \mathrm{mg} / \mathrm{m}^{\wedge} 2\right)$ daily and BEV $(10 \mathrm{mg} / \mathrm{kg}$ every two weeks) administered during RT for six weeks. One month after completion of RT, adjuvant TMZ $\left(150 \mathrm{mg} / \mathrm{m}^{\wedge} 2 \times 5\right.$ days $)$ was delivered monthly with BEV $(10 \mathrm{mg} / \mathrm{kg})$ every two weeks until progression, toxicities, or 12 months total. The standard regimen involved RT to 60 Gy with concurrent TMZ $\left(75 \mathrm{mg} / \mathrm{m}^{\wedge} 2\right)$ given daily during RT for six weeks. One month after completion of RT, TMZ (150 mg/m^ $2 \times 5$ days) was delivered monthly for up to 12 months. At the time of disease progression, all patients were offered BEV infusion every three weeks as salvage therapy. A total of 19 patients received salvage BEV after progression on the standard arm.

All patients received DXM during the perioperative period which was weaned prior to the start of RT if possible. We categorized patients based on the use of DXM during RT for this analysis. Patients who could not taper off or who restarted DXM during RT were categorized as positive for steroid usage.

Progression free time refers to the interval between the diagnosis and progression. Progression was defined as: (1) New T1 and gadolinium enhancement; (2) T2 FLAIR progression; (3) new or worsening neurological symptoms; (4) a change in therapy; or (5) death. For patients receiving $\mathrm{BEV}$, universally agreed criteria were 
used to determine progression [17]. The MRIs were independently reviewed by a neuroradiologist. The overall survival time was defined as the interval between diagnosis and death from any cause.

Descriptive analyses were conducted to estimate patient clinical and demographic characteristics. Kaplan-Meier analysis was performed to assess differences in event-free (progression and death) experience between groups using all patients as well as between groups using subsets of patients (eg. those only on TMZ). The log-rank test was invoked to assess statistical significance in univariable comparisons [18]. To adjust for potential covariate effects, Cox proportional hazards regression was used as the modeling paradigm [19]. Multivariate analysis was conducted using Cox regression modeling. The proportional hazards assumption was assessed both visually using plots of the observed standardized score process with 20 simulated realizations for each covariate included in the model as well as quantitatively using the Kolmogorov-type supremum test based on 1000 simulations [20].

\section{Results}

Whole cohort observations: univariable analysis

A total of 73 patients were treated during this study with an age range at diagnosis between 28 and 79 years (median age 61 years) (Table 1). With a median follow-up of 15.6 months, $67(91.8 \%)$ patients were deceased. The average age at death was 60.5 years (age range $30-81$ years). Fortyfour patients $(60 \%)$ were male; $29(40 \%)$ were female. Two patients died before completion of RT due to progression after $6 \mathrm{~Gy}$ and $26 \mathrm{~Gy}$, respectively. One patient developed a wound infection during radiation and received only $16 \mathrm{~Gy}$. The median RT dose was 60 Gy. The majority (38; $52 \%$ ) of patients underwent a GTR, while $24(33 \%)$ underwent a STR and 11 (15\%) had a biopsy. Table 1 provides a description of this cohort of patients in terms of extent of resection, the concurrent regimen used, smoking, hypertension (HTN), body mass index (BMI), diabetes mellitus (DM), hyperlipidemia, and the use of DXM during RT. The OS for the whole cohort was 15.9 months (Fig. 1a), while the PFS was 7.7 months. The one-year and two-year survival was 70 and $31 \%$, respectively. The extent of resection was a prognostic indicator for OS ( $p=.0044)$ (Fig. 1b). The median survival following GTR was 22.6 months, STR was 14.9 months, and biopsy was 12.1 months. Patients who underwent a biopsy for GBM had a poor prognosis for OS. The addition of BEV to TMZ with RT was borderline significantly associated with increased PFS (9.4 vs. 5.1 months, $p=0.0574)$ although was not significantly associated with OS (18.1 vs. 15.3 months respectively, $p=0.3064$ ).

\section{Influence of dexamethasone: univariable analysis}

DXM use concurrent with RT was a poor prognostic indicator of both OS (median 12.7 vs. 22.5 months, $p=0.02$,
Table 1 Population statistics of patients with newly diagnosed GBM

\begin{tabular}{|c|c|c|c|}
\hline Population statistics & Category & Absolute & Percentage \\
\hline $\begin{array}{l}\text { Median age } \\
\text { at diagnosis }\end{array}$ & & 61.0 & \\
\hline $\begin{array}{l}\text { Average age } \\
\text { at death }\end{array}$ & & 60.5 & \\
\hline \multirow[t]{2}{*}{ Gender } & M & 44 & $60 \%$ \\
\hline & $\mathrm{F}$ & 29 & $40 \%$ \\
\hline \multirow[t]{3}{*}{ Extent of resection } & GTR & 38 & $52 \%$ \\
\hline & STR & 24 & $33 \%$ \\
\hline & Biopsy & 11 & $15 \%$ \\
\hline Median RT dose & & 6000 & \\
\hline \multirow[t]{2}{*}{ Concurrent agents } & Temozolomide alone & 39 & $53 \%$ \\
\hline & $\begin{array}{l}\text { Temozolomide and } \\
\text { Bevacizumab }\end{array}$ & 34 & $47 \%$ \\
\hline \multirow[t]{2}{*}{ Smoker } & No & 43 & $60 \%$ \\
\hline & Yes & 29 & $40 \%$ \\
\hline \multirow[t]{2}{*}{ HTN } & No & 32 & $44 \%$ \\
\hline & Yes & 41 & $56 \%$ \\
\hline \multirow[t]{3}{*}{ BMI } & Normal $(<25)$ & 17 & $23 \%$ \\
\hline & Overweight (25-30) & 37 & $51 \%$ \\
\hline & Obese $(>30)$ & 19 & $26 \%$ \\
\hline \multirow[t]{2}{*}{ DM } & No & 63 & $86 \%$ \\
\hline & Yes & 10 & $14 \%$ \\
\hline \multirow[t]{2}{*}{ Hyperlipidemia } & No & 46 & $63 \%$ \\
\hline & Yes & 27 & $37 \%$ \\
\hline \multirow{2}{*}{$\begin{array}{l}\text { Dexamethasone } \\
\text { during RT }\end{array}$} & No & 37 & $51 \%$ \\
\hline & Yes & 36 & $49 \%$ \\
\hline
\end{tabular}

Fig. 1c) and PFS (median 6.0 vs. 8.8 months, $p=0.002$ ) in the whole cohort. Given the known significance of the extent of resection on survival, we analyzed the effect of DXM in patients with either a GTR (Fig. 1d) or STR/Biopsy. For patients who underwent GTR, those who were treated with DXM had a median survival of 16.3 months compared to 24.6 months without DXM $(p=0.15)$. Patients who underwent either STR or biopsy had nearly a two-fold reduction of median survival with DXM administration (8.2 months) compared to those without DXM (15.3 months) although statistical significance was not reached $(p=0.20)$.

Since BEV has been shown to reduce the use of DXM, we conducted additional analyses in patients who received TMZ only during RT without BEV. In patients receiving TMZ, DXM use concurrent with RT was a poor prognostic indicator of both OS (12.7 vs. 22.6 months, $p=0.003$, Fig. 2a) and PFS (3.6 vs. 8.4 months, $p<0.0001)$. Even in patients who underwent a GTR treated with TMZ alone, DXM use was a poor 
A

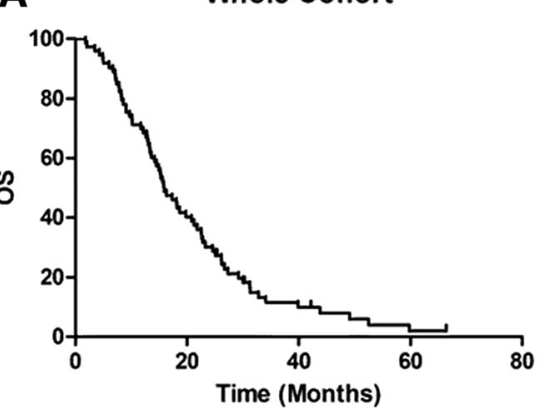

B

Extent of Resection

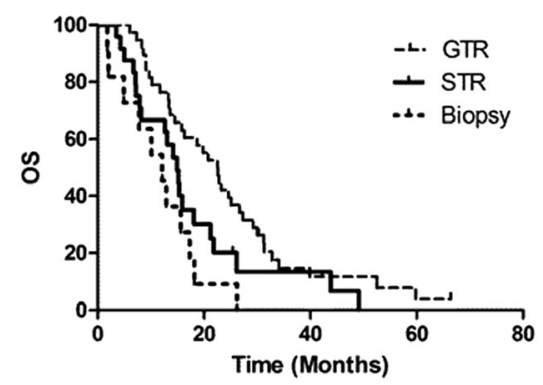

C

\%

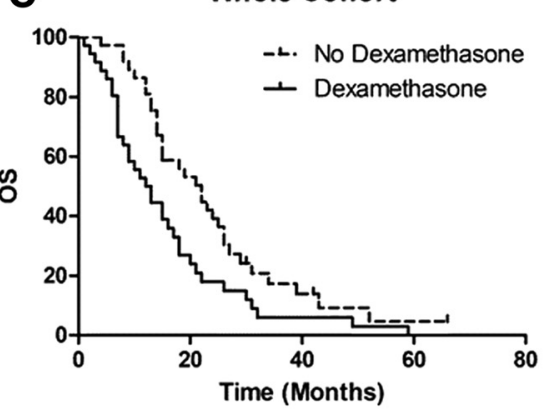

D

GTR Only

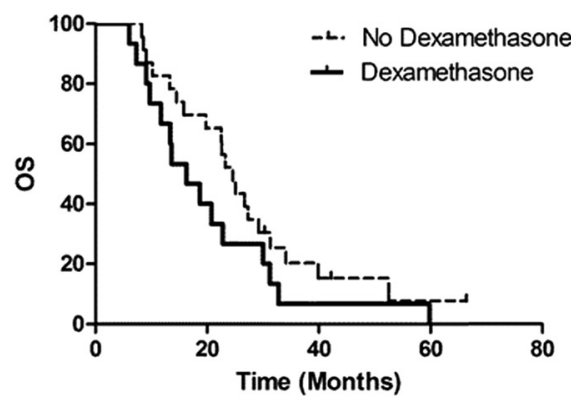

Fig. 1 Kaplan-Meier curve shows the (a) OS of the whole cohort of newly diagnosed GBM patients after surgical resection, with a median of 15.9 months. b The extent of resection correlated with OS: GTR 22.6 months, STR 14.9 months, and biopsy 12.1 months. c DXM use concurrent with RT was a poor prognostic indicator of OS (median 12.7 vs. 22.5 months, $p=0.02$ by log-rank test). $\mathbf{d}$ For patients who underwent GTR, those who were treated with DXM had a median survival of 16.3 months compared to 24.6 months without DXM ( $p=0.15$ by log-rank test)

prognostic factor for OS (13.5 vs 25.1 months, $p=0.01$, Fig. 2b). Interestingly, DXM did not reduce OS in patients who received TMZ and BEV concurrently with RT (22.9 vs 22.8 months, $p=0.4818$, Fig. 2 c). DXM did not affect PFS in patients receiving concurrent BEV with TMZ (9.5 vs. 8.9 months, $p=0.6520$ ).

\section{Multivariable analysis}

Cox proportional hazards regression modeling was used to assess whether DXM administration continued to confer unfavorable time-to-event (progression and death) as seen in the univariable analyses. Two separate sets of multivariable models were run to supplement the respective univariable analyses. One set included all patients in the observation cohort. The second set included those administered only TMZ. In the first set of analyses (on the whole cohort), models for OS and PFS included the following covariate list: DXM, BEV, extent of resection, age at diagnosis, gender, XRT dosage, smoking status, and BMI. For the whole cohort analysis, significant predictors of unfavorable OS included patients with DXM (hazard ratio $(\mathrm{HR})=1.72, p=0.045)$ and decreasing XRT dosage (a $1 \mathrm{sd}$ decrease of 7.41 Gy results in $\mathrm{HR}=1.66, p=0.0035)$. Extent of resection was borderline significant (Wald chisquare $p=0.0617$ ) for OS. Significant predictors of unfavorable PFS included patients with DXM $(\mathrm{HR}=2.98$, $p=0.0004)$ as well as patients without $\mathrm{BEV}(\mathrm{HR}=2.64$, $p=0.0015)$. Results using only those administered TMZ were similar for OS to the whole cohort analysis: DXM use resulted in an increase in hazard of death $(\mathrm{HR}=2.87$, $p=0.0255)$ while decreasing XRT dosage by 1 sd (7.41 Gy) resulted in an increase in hazard of death $(\mathrm{HR}=1.63, p=0.0079)$. For PFS, DXM use was the only covariate found to be significantly associated with less favorable outcome $(\mathrm{HR}=4.46, p=0.0015)$.

There were no evidences for departure from the proportional hazards assumption for any of the covariates included in the models when inspecting the diagnostic plots of the observed standardized model process plotted over time. Additionally, all $p$-values associated with the Kolmogorov-type supremum test, which quantitatively tests for departures from proportional hazards, were not significant.

\section{Discussion}

Several studies have investigated the combination of BEV and TMZ with RT in the treatment of newly diagnosed GBM [1, 21-27]. Our results compare favorably to these previous studies as the median OS, PFS, and one- and two-year survival data for patients with GBM who have been treated with a combination of RT and chemotherapy following initial resection are presented in 
A

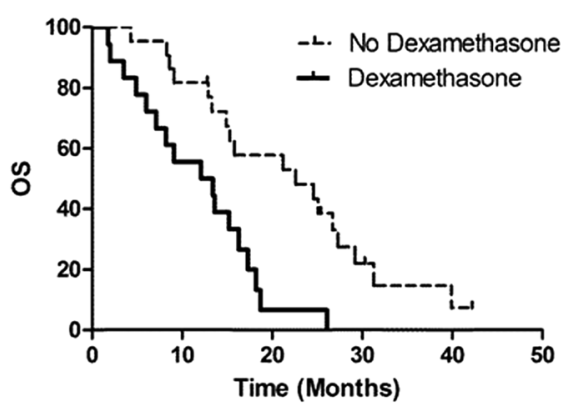

B

Temozolomide and GTR

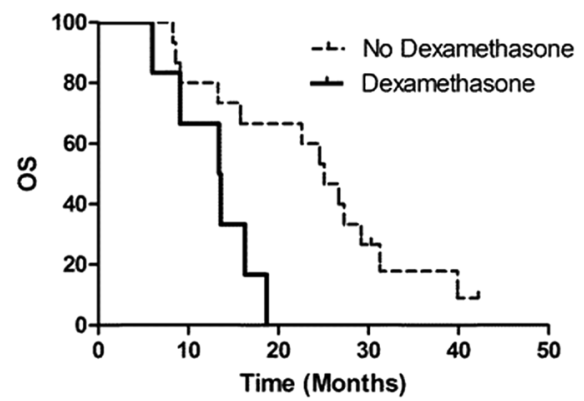

\section{Temozolomide and Bevacizumab}

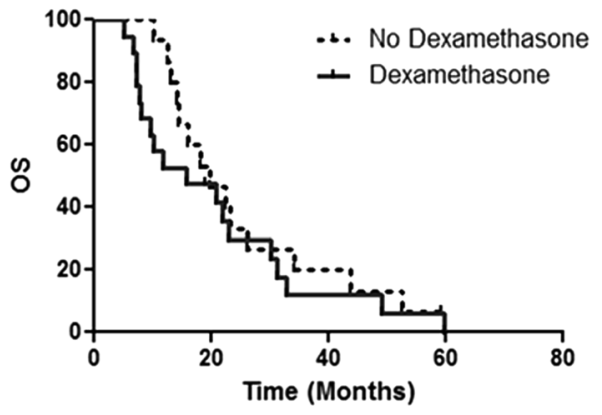
months, $p=0.003$ by log-rank test). b DXM use was a poor prognostic factor for OS in patients who underwent a GTR treated with TMZ alone (13.5 vs 25.1 months, $p=0.01$ by log-rank test). c DXM did not reduce OS patients who received TMZ and BEV concurrent with RT (22.9 vs 22.8 months, $p=0.4818$ by log-rank test)

Table 2. Our results with TMZ and RT are similar to previous studies in terms of PFS and OS. The OS for our whole cohort was 15.9 months, while the PFS was 7.4 months. The one-year and two-year survival in our study was 71 and $32 \%$, respectively. Concurrent BEV and TMZ with RT was significantly associated with increased PFS although was not significantly associated with OS. Our study also confirms the known literature that the extent of resection was a significant prognostic indicator for OS. The median survival following GTR was 22.5 months, STR was 14.93 months, and biopsy was 12.1 months.

In Keth et al.'s study of 345 patients with newly diagnosed GBM, they demonstrated that GTR was a favorable prognostic factor for OS while the value of incomplete resection remained questionable [28]. They recommended biopsy only if GTR could not be safely performed as biopsy provided acceptable histological diagnosis and determination of MGMT promoter methylation status [28].

Table 2 Survival for patients with GBM following radiation with biological agents

\begin{tabular}{|c|c|c|c|c|c|}
\hline Study & Treatment & Median OS & Median PFS & One-year survival & Two-year survival \\
\hline Current study (2015) $(n=73)$ & RT + BEV + TMZ (12 cycles) & 15.9 months & 7.7 months & $70 \%$ & $31 \%$ \\
\hline Stupp [1] $(n=287)$ & $R T+T M Z$ & 14.6 months & 6.9 months & $61 \%$ & $27 \%$ \\
\hline Grossman [19] $(n=244)$ & $\mathrm{RT}+\mathrm{TMZ}+$ novel agents & 19.6 months & - & $81 \%$ & $37 \%$ \\
\hline Lai [21] $(n=70)$ & $\mathrm{RT}+\mathrm{BEV}+\mathrm{TMZ}$ & 19.6 months & 13.6 months & - & - \\
\hline Vredenburgh [24] $(n=75)$ & $\mathrm{RT}+\mathrm{BEV}+\mathrm{TMZ}+$ Irinotecan & 21.2 months & 14.2 months & $78.7 \%$ & $44.9 \%$ \\
\hline Narayana [22] $(n=51)$ & $\mathrm{RT}+\mathrm{BEV}+\mathrm{TMZ}$ (six cycles) & 23 months & 13 months & $85 \%$ & $43 \%$ \\
\hline
\end{tabular}

OS Overall survival

PFS Progression-free survival

$R T$ Radiotherapy

BEV Bevacizumab

TMZ Temozolomide 
Two eagerly anticipated phase III trials (Avaglio and RTOG 0825) have focused on the upfront use of BEV with TMZ and RT in newly-diagnosed GBM [14-16, 29]. Both of these studies showed significantly prolonged PFS and similar adverse effects of BEV, however, the interim analysis of OS did not reach statistical significance [14-16, 30, 31]. The Avaglio study demonstrated maintenance of quality of life outcomes, while RTOG 0825 showed a decreased quality of life [14, 30,31]. OS results from these two trials do not support routine upfront BEV. Questions remain whether BEV should be used to treat patients with recurrent GBM since it offers QOL and PFS benefits [31].

The Avaglio trial addressed the use of glucocorticoids in patients who were treated with BEV [29]. Of the patients who received DXM at baseline, DXM was discontinued in $66.3 \%$ of patients who received BEV compared to $47.1 \%$ of patients receiving placebo. Furthermore, of the patients who were not treated with DXM at baseline, the time to initiation of DXM was significantly longer with BEV than placebo [29]. Both of these observations support the hypothesis that BEV reduces cerebral edema to improve clinical symptoms.

DXM is commonly used in the treatment of GBM to manage cerebral edema and has anti-inflammatory properties [11, 32]. It is often prescribed at diagnosis to decrease tumor-associated vasogenic edema and improve symptoms [13]. DXM is often continued after biopsy or resection to reduce post-operative edema and then continued during RT to decrease radiation-associated edema [13]. DXM is occasionally utilized concurrently with TMZ and RT after the initial surgical resection of a GBM. It has been shown that DXM works as an antagonist on TMZ-induced apoptosis in human glioblastoma T98G cells, suggesting that the combination of TMZ and DXM may be counteractive in treating GBM [11].

Due to the toxicities associated with DXM, including hyperglycemia, myopathy, osteoporosis, and immunosuppression, many patients with GBM decrease or discontinue their DXM use early in their management of GBM $[12,13]$. Contrarily, other patients may necessitate an increase in DXM if they experience focal neurologic symptoms (hemiparesis, visual field deficit, or aphasia) or global symptoms (headache, nausea, or decreased appetite) [13].

Cerebral edema is a common side effect of chemoradiotherapy for GBM, necessitating glucocorticoid management [33]. Substantial deregulation of blood glucose levels may ensue. In their study of 106 GBM patients, Mayer et al. determined that one or more deregulated blood glucose values $>10 \mathrm{mM}$ was associated with a reduction in median OS from 16.7 to 8.8 months [33]. In addition, a significantly poorer OS was found in patients with hyperglycemia who underwent complete tumor resection.
Several reports have demonstrated DXM dependency during RT as an independent poor predictor of survival in GBM [32, 34, 35]. In their study of 173 patients with malignant gliomas, Watne et al. reported that patients who were corticosteroid-dependent after craniotomy had a 1.9 relative death risk as compared to patients who were off steroids post-operatively [35]. They did not examine whether the increased relative risk of death associated with corticosteroid use was influenced by the extent of resection. Michaelsen et al. reported that the use of corticosteroid therapy was significantly correlated with patient survival and disease progression in their study of 225 GBM patients [34]. Corticosteroid therapy at TMZ and RT initiation had a significant negative impact on OS $(p<0.0001)$. This study examined the interaction of age with corticosteroid use but did not investigate the interaction of extent of resection and corticosteroid use. Patients with a GTR would be expected to have the longest OS. These data together demonstrated that DXM administration produces shorter overall survival even without measurable contrast-enhancing tumor, suggesting the effect is independent of the extent of resection. We found by both univariate and multivariable analysis that DXM use correlated with decreased OS. Thus, our data both reinforce previous work as well as expand the conclusions to include the extent of resection along with DXM use.

Gorlia et al. analyzed the data from 300 patients with recurrent GBM in eight phase I or II trials conducted by the EORTC Brain Tumor Group [36]. A total of 138 patients received TMZ and RT followed by TMZ as first-line therapy, while 158 patients received RT alone or with another chemotherapy; four were treated without previous radiotherapy [36]. They demonstrated that patients who received TMZ and RT followed by TMZ were significantly less often treated with baseline steroids $(57 \%$ vs $73 \%, p=0.004)$. They concluded that patients treated with steroids at baseline had a shorter OS. Furthermore, they suggested that the use of antiangiogenic therapies may change the prognostic potential of certain factors, such as BEV administration may reduce the detrimental effect of the need for steroids, at least on PFS [36].

Vredenburgh et al. investigated the effect of corticosteroid use in GBM patients at first or second recurrence treated with BEV in the BRAIN study [12]. The BRAIN study was a phase II, multicenter, randomized trial of BEV alone or concurrent with irinotecan (CPT-11) in patients with recurrent GBM. The authors reported that the majority of patients who had an objective response or PFS $>6$ months experience corticosteroid dose reduction. They suggested that BEV may have a corticosteroid-sparing effect in patients with recurrent GBM and that a reduction in corticosteroids may positively affect patient QOL [12]. 
Our present study concurs with previous studies that DXM use concurrent with RT is a significantly poor prognostic indicator of both PFS and OS in patients with GBM. Our study is the first in the literature, to our knowledge, that investigated upfront DXM use concurrently with RT, TMZ, and BEV. In patients who received TMZ, DXM use concurrent with RT was a poor prognostic indicator of both OS and PFS. However, DXM did not reduce OS or affect PFS in patients who received TMZ and BEV concurrently with RT. In addition, of all of the patients treated with DXM and RT, the OS was significantly shorter in patients who only received TMZ compared to those who received both TMZ and BEV. Our results suggest that it is not recommended to treat GBM patients with upfront DXM or with DXM concurrent with TMZ. However, if DXM is necessary due to the negative side effects of GBM such as headaches, then the administration of BEV concurrent with DXM may be beneficial. We have shown that DXM use during RT with concurrent TMZ correlated with reduced OS and PFS unless BEV was administered.

\section{Conclusion}

The combination therapy of TMZ, BEV, and RT was safe and well tolerated in this study. Similar to previous reports in the literature, RT with concurrent BEV and TMZ was significantly associated with increased PFS but not OS. The present study demonstrates that DMX use concurrent with RT and TMZ was a poor prognostic indicator of both OS and PFS. Contrarily, DMX did not reduce OS in patients who received TMZ and BEV concurrently with RT. Upfront use of BEV may prove beneficial in GBM patients who require DXM use and are treated with concurrent TMZ and RT.

\section{Competing interests}

The authors declare that they have no competing interests.

\section{Authors' contributions}

LBES performed the literature search, analyzed the primary data, and played the primary role in the writing of the manuscript. BJS, AS, and LC verified the univariable analyses and performed all multivariable statistical analyses using Cox regression and wrote portions of the manuscript (the statistical portion of the Methods section and parts of the Results section). DAS was the neurosurgeon who performed the surgical resection and analyzed the primary data. SP was the behavioral oncologist who ensured compliance with the treatment protocol and contributed to the writing of the manuscript. TDB was the neuropathologist who performed the pathology studies. RR was the medical neurooncologist who administered the chemotherapy to the patients and participated in the writing of the manuscript. ACS was the radiation oncologist who designed the study, collected and analyzed the primary data, administered the radiation to the patients, and wrote the manuscript. All authors read and approved the final manuscript.

\section{Acknowledgments}

This work was supported by funding from NCl grant P30 CA177558 and from a Norton Healthcare Foundation grant.

\section{Author details}

${ }^{1}$ Norton Neuroscience Institute, Norton Healthcare, Louisville, KY, USA. ${ }^{2}$ The Brain Tumor Center, Norton Healthcare, Louisville, KY, USA. ${ }^{3}$ The Markey Cancer Center, University of Kentucky School of Medicine, Lexington, KY, USA. ${ }^{4}$ The Norton Cancer Institute, Norton Healthcare, Louisville, KY, USA. ${ }^{5}$ The Norton Cancer Institute Radiation Center, 676 S. Floyd St., Suite 100, Louisville, KY 40202, USA.

Received: 13 May 2015 Accepted: 19 October 2015

Published online: 31 October 2015

\section{References}

1. Stupp R, Mason WP, van den Bent MJ, Weller M, Fisher B, Taphoorn MJ, et al. Radiotherapy plus concomitant and adjuvant temozolomide for glioblastoma. N Engl J Med. 2005;352(10):987-96.

2. Albert FK, Forsting M, Sartor K, Adams HP, Kunze S. Early postoperative magnetic resonance imaging after resection of malignant glioma: objective evaluation of residual tumor and its influence on regrowth and prognosis. Neurosurgery. 1994;34(1):45-60.

3. Fine HA, Dear KB, Loeffler JS, Black PM, Canellos GP. Meta-analysis of radiation therapy with and without adjuvant chemotherapy for malignant gliomas in adults. Cancer. 1993;71(8):2585-97.

4. Lacroix M, Abi-Said D, Fourney DR, Gokaslan ZL, Shi W, DeMonte F, et al. A multivariate analysis of 416 patients with glioblastoma multiforme: prognosis, extent of resection, and survival. J Neurosurg. 2001;95(2):190-8.

5. Wong ET, Hess KR, Gleason MJ, Jaeckle KA, Kyritsis AP, Prados MD, et al. Outcomes and prognostic factors in recurrent glioma patients enrolled onto phase II clinical trials. J Clin Oncol. 1999;17(8):2572-8.

6. Ronning PA, Helseth E, Meling TR, Johannesen TB. A population-based study on the effect of temozolomide in the treatment of glioblastoma multiforme. Neuro Oncol. 2012;14(9):1178-84.

7. Stupp R, Dietrich PY, Ostermann KS, Pica A, Maillard I, Maeder P, et al. Promising survival for patients with newly diagnosed glioblastoma multiforme treated with concomitant radiation plus temozolomide followed by adjuvant temozolomide. J Clin Oncol. 2002;20(5):1375-82.

8. Stupp R, Hegi ME, Mason WP, van den Bent MJ, Taphoorn MJ, Janzer RC, et al. Effects of radiotherapy with concomitant and adjuvant temozolomide versus radiotherapy alone on survival in glioblastoma in a randomised phase III study: 5-year analysis of the EORTC-NCIC trial. Lancet Oncol. 2009;10(5):459-66.

9. Szczepanek D, Marchel A, Moskala M, Krupa M, Kunert P, Trojanowski T. Efficacy of concomitant and adjuvant temozolomide in glioblastoma treatment. A multicentre randomized study. Neurol Neurochir Pol. 2013;47(2):101-8.

10. Knizhnik AV, Roos WP, Nikolova T, Quiros S, Tomaszowski KH, Christmann M, et al. Survival and death strategies in glioma cells: autophagy, senescence and apoptosis triggered by a single type of temozolomide-induced DNA damage. PLoS ONE. 2013;8(1):e55665.

11. Sur P, Sribnick EA, Patel SJ, Ray SK, Banik NL. Dexamethasone decreases temozolomide-induced apoptosis in human gliobastoma T98G cells. Glia. 2005;50(2):160-7.

12. Vredenburgh JJ, Cloughesy T, Samant M, Prados M, Wen PY, Mikkelsen T, et al. Corticosteroid use in patients with glioblastoma at first or second relapse treated with bevacizumab in the BRAIN study. Oncologist. 2010;15(12):1329-34.

13. Deutsch MB, Panageas KS, Lassman AB, DeAngelis LM. Steroid management in newly diagnosed glioblastoma. J Neurooncol. 2013;113(1):111-6.

14. Chinot O, Wick W, Mason W, Henriksson R, Saran F, Nishikawa R, et al. Phase III trial of bevacizumab added to standard radiotherapy and temozolomide for newly-diagnosed glioblastoma: mature progression-free survival and preliminary overall survival results in Avaglio [Abstract]. Neuro-Oncology. 2012;14(6):101

15. Chinot OL, de La Motte RT, Moore N, Zeaiter A, Das A, Phillips H, et al. AVAglio: Phase 3 trial of bevacizumab plus temozolomide and radiotherapy in newly diagnosed glioblastoma multiforme. Adv Ther. 2011;28(4):334-40.

16. Gilbert MR, Dignam J, Won M. RTOG: 0825: Phase III double-blind placebocontrolled trial evaluating bevacizumab (Bev) in patients (Pts) with newly diagnosed glioblastoma (GBM). J Clin Oncol 2013, 31(Suppl(abstr 1)).

17. Wen PY, Macdonald DR, Reardon DA, Cloughesy TF, Sorensen AG, Galanis E, et al. Updated response assessment criteria for high-grade gliomas: 
response assessment in neuro-oncology working group. J Clin Oncol. 2010;28(11):1963-72.

18. Kaplan EL, Meier P. Nonparametric estimation from incomplete observations. J Am Stat Assoc. 1958;53:457-81.

19. Cox DR. Regression models and life-tables. J R Statist Soc B. 1972;34(2):187-220.

20. SAS Institute Inc. SAS/STAT 9.2 User's Guide. 2008.

21. Grossman SA, Ye X, Piantadosi S, Desideri S, Nabors LB, Rosenfeld M, et al. Survival of patients with newly diagnosed glioblastoma treated with radiation and temozolomide in research studies in the United States. Clin Cancer Res. 2010;16(8):2443-9.

22. Lai A, Filka E, McGibbon B, Nghiemphu PL, Graham C, Yong WH, et al. Phase Il pilot study of bevacizumab in combination with temozolomide and regional radiation therapy for up-front treatment of patients with newly diagnosed glioblastoma multiforme: interim analysis of safety and tolerability. Int J Radiat Oncol Biol Phys. 2008;71(5):1372-80.

23. Lai A, Tran A, Nghiemphu PL, Pope WB, Solis OE, Selch M, et al. Phase II study of bevacizumab plus temozolomide during and after radiation therapy for patients with newly diagnosed glioblastoma multiforme. J Clin Oncol. 2011;29(2):142-8.

24. Narayana A, Gruber D, Kunnakkat S, Golfinos JG, Parker E, Raza S, et al. A clinical trial of bevacizumab, temozolomide, and radiation for newly diagnosed glioblastoma. J Neurosurg. 2012;116(2):341-5.

25. Vredenburgh JJ, Desjardins A, Kirkpatrick JP, Reardon DA, Peters KB, Herndon JE et al. Addition of bevacizumab to standard radiation therapy and daily temozolomide is associated with minimal toxicity in newly diagnosed glioblastoma multiforme. Int J Radiat Oncol Bio Phys 2012;82(1):58-66.

26. Vredenburgh JJ, Desjardins A, Reardon DA, Peters KB, Herndon JE, Marcello J, et al. The addition of bevacizumab to standard radiation therapy and temozolomide followed by bevacizumab, temozolomide, and irinotecan for newly diagnosed glioblastoma. Clin Cancer Res. 2011;17(12):4119-24.

27. Vredenburgh JJ, Desjardins A, Kirkpatrick JP, Reardon DA, Peters KB, Herndon JE, et al. Addition of bevacizumab to standard radiation therapy and daily temozolomide is associated with minimal toxicity in newly diagnosed glioblastoma multiforme. Int J Radiat Oncol Biol Phys. 2012;82(1):58-66

28. Kreth FW, Thon N, Simon M, Westphal M, Schackert G, Nikkhah G, et al. Gross total but not incomplete resection of glioblastoma prolongs survival in the era of radiochemotherapy. Ann Oncol. 2013;24(12):3117-23.

29. Chinot OL, Wick W, Mason W, Henriksson R, Saran F, Nishikawa R, et al. Bevacizumab plus radiotherapy-temozolomide for newly diagnosed glioblastoma. N Engl J Med. 2014;370(8):709-22.

30. Haines IE, Gabor Miklos GL. Bevacizumab for newly diagnosed glioblastoma. N Engl J Med. 2014;370(21):2048.

31. Weller M, Yung WK. Angiogenesis inhibition for glioblastoma at the edge: beyond AVAGlio and RTOG 0825. Neuro Oncol. 2013;15(8):971.

32. Hohwieler SM, Freidberg SR, Heatley GJ, Lo TC. Glucocorticoid dependency as a prognostic factor in radiotherapy for cerebral gliomas. Acta Oncol. 1989;28(1):51-5.

33. Mayer A, Vaupel $P$, Struss HG, Giese A, Stockinger M, Schmidberger $H$. Strong adverse prognostic impact of hyperglycemic episodes during adjuvant chemoradiotherapy of glioblastoma multiforme. Strahlenther Onkol. 2014;190(10):933-8.

34. Michaelsen SR, Christensen IJ, Grunnet K, Stockhausen MT, Broholm H, Kosteljanetz $\mathrm{M}$, et al. Clinical variables serve as prognostic factors in a model for survival from glioblastoma multiforme: an observational study of a cohort of consecutive non-selected patients from a single institution. BMC Cancer. 2013;13:402.

35. Watne $\mathrm{K}$, Hannisdal $\mathrm{E}$, Nome $\mathrm{O}$, Hager $\mathrm{B}$, Hirschberg $\mathrm{H}$. Prognostic factors in malignant gliomas with special reference to intra-arterial chemotherapy. Acta Oncol. 1993;32(3):307-10.

36. Gorlia T, Stupp R, Brandes AA, Rampling RR, Fumoleau P, Dittrich C, et al. New prognostic factors and calculators for outcome prediction in patients with recurrent glioblastoma: a pooled analysis of EORTC Brain Tumour Group phase I and II clinical trials. Eur J Cancer. 2012;48(8):1176-84.

\section{Submit your next manuscript to BioMed Central and take full advantage of:}

- Convenient online submission

- Thorough peer review

- No space constraints or color figure charges

- Immediate publication on acceptance

- Inclusion in PubMed, CAS, Scopus and Google Scholar

- Research which is freely available for redistribution

Submit your manuscript at www.biomedcentral.com/submit 